\title{
Microstructural studies of laser irradiated graphite surfaces ${ }^{\text {a) }}$
}

\author{
J. S. Speck \\ Department of Materials Science and Engineering, Massachusetts Institute of Technology, \\ Cambridge, Massachusetts 02139 \\ J. Steinbeck ${ }^{\text {b) }}$ \\ Department of Physics, Massachusetts Institute of Technology, Cambridge, Massachusetts 02139
}

\begin{abstract}
M.S. Dresselhaus
Department of Physics and Department of Electrical Engineering and Computer Science, Massachusetts Institute of Technology, Cambridge, Massachusetts 02139
\end{abstract}

(Received 30 October 1989; accepted 2 February 1990)

\begin{abstract}
The structure of pulsed laser irradiated graphite surfaces has been elucidated. The pulse fluences range up to $4 \mathrm{~J} \mathrm{~cm}^{-2}$ with durations no longer than $30 \mathrm{~ns}$. The region exterior to the irradiated spot is littered with $\sim 0.1 \mu \mathrm{m}$ diameter carbon spheroids. The boundary region is characterized by both spheroids and torn layers $1-5 \mu \mathrm{m}$ in lateral extent. The central region displays carbon spheroids and surface upheavals. The carbon spheroids are attributed to hydrodynamic sputtering of carbon. The surface upheavals and torn carbon layers are attributed to constrained thermal expansion and contraction of the irradiated region. It is estimated that a nearly instantaneous $1000{ }^{\circ} \mathrm{C}$ temperature change is necessary to cause the observed surface deformation. Pulse fluences in excess of $0.8 \mathrm{~J} \mathrm{~cm}^{-2}$ cause a thin layer of carbon to melt. This is proven by the fact that the irradiated layer in the solid phase has a turbostratic structure. Electron diffraction experiments and dark-field imaging experiments show that the basal plane grain size of the resolidified material varies from $\sim 20 \AA$ at the melt threshold to $\sim 100 \AA$ for samples irradiated with $4.0 \mathrm{~J} \mathrm{~cm}^{-2}$.
\end{abstract}

\section{INTRODUCTION}

The high temperature phases and properties of carbon, the most refractory material known to man, have long been of interest. The nature of the high temperature-low pressure region of the carbon phase diagram is still known with little certainty. ${ }^{1}$ The high melting temperature of carbon (estimated to be $-4500 \mathrm{~K}^{2}$ ) and the high vapor pressure near the melting point prove to be substantial experimental barriers to effectively probing this region of the P-T phase diagram. It is difficult to confine a $\sim 4500 \mathrm{~K}$ melt pool of carbon (what could be used for a crucible?). Hence most direct experimental work on the structure and properties of liquid carbon have involved rapid heating of carbon either by electrical currents ${ }^{3,4}$ or by short intense laser pulses. ${ }^{5-7}$

Previous work by Braunstein et al. on the problem using Rutherford backscattering spectrometry (RBS), Raman spectroscopy, and ion channeling has led to values of the disordered layer thickness as a function of laser pulse fluence, ${ }^{8}$ as shown in Fig. 1. These investigators concluded that liquid carbon was formed when

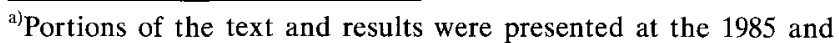
1987 Fall Meetings of the Materials Research Society.

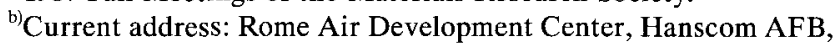
Massachusetts 01731.
}

samples were irradiated by a $30 \mathrm{~ns}$ pulsed ruby laser $(\lambda=6943 \AA)$ with fluences greater than $0.6 \mathrm{~J} \mathrm{~cm}^{-2}$. Further evidence for melting can be obtained by direct microscopic examination of the irradiated region and then drawing conclusions from the observed structure.

In this paper we report not on the high temperature phases of carbon but rather on the structural characteristics of the post-irradiated carbon surfaces. We will show that the post-irradiated material is a fine-grained turbostratic structure (random rotations and translations between successive carbon sheets) consistent with rapid solidification of a pool of liquid carbon.

\section{EXPERIMENTAL PROCEDURE}

Samples of pristine highly oriented pyrolytic graphite (HOPG) were irradiated using one of two systems: a 30 ns pulsed ruby laser $(\lambda=6943 \AA)$ passed through a quartz homogenizer for spatial uniformity, or a $20 \mathrm{ps}$. pulsed frequency doubled $\mathrm{Nd}$ : $\operatorname{YAG}(\lambda=5320 \AA)$ laser. The incident radiation was normal to the sample surface. The incident beam was unpolarized and had a spot size on the sample of $\sim 3-5 \mathrm{~mm}$. All samples were irradiated by the ruby and Nd: YAG lasers in air. SEM was performed using a JEOL $200 \mathrm{CX}$ microscope operating at $80 \mathrm{kV}$. TEM was also performed using a JEOL $200 \mathrm{CX}$ microscope operating at $200 \mathrm{kV}$. Bright-field TEM, selected area diffraction, and high resolution 


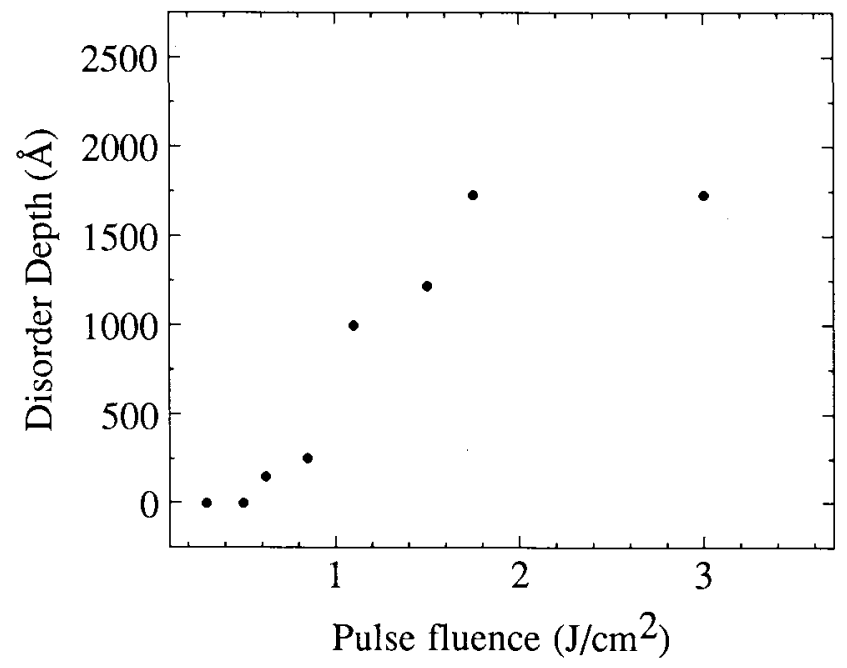

FIG. 1. Effect of laser pulse fluence from a $30 \mathrm{~ns}$ pulse ruby laser $(\lambda=6943 \AA$ ) on disorder depth as determined by ion channeling. (Taken from Braunstein et $a l^{8}{ }^{8}$ )

TEM were performed. Chemical analysis, by $\mathrm{x}$-ray fluorescence, was performed on a VG HB5 STEM. Specimens for TEM and STEM were prepared by peeling the HOPG from the unirradiated side of the specimen down to an electron transparent thickness $(\sim 1000 \AA)$ and then repeatedly bathing, $\sim 20$ times, the thin specimen in either methanol or acetone.

\section{RESULTS}

Any single technique for evaluating a complex structure will provide only limited information. By using several different microstructural characterization techniques, a deeper understanding of a structure can be developed. This will be shown to be the case for the laser-irradiated samples discussed below.

For samples irradiated with the ruby laser using energy fluences less than $0.6 \mathrm{~J} \mathrm{~cm}^{-2}$, SEM and optical microscopy results indicate no alteration of the surface structure of the HOPG. Channeling measurements performed by Venkatesan et al. ${ }^{5}$ show that pulse fluences in excess of $0.6 \mathrm{~J} \mathrm{~cm}^{-2}$ are necessary to form a disordered surface layer. Hence, if melting does occur at these low laser energy fluences, then the melt solidifies in a 'quiet', uneventful, and, most importantly, epitaxial manner.

For laser energy fluences in excess of $1.0 \mathrm{~J} \mathrm{~cm}^{-2}$, the structure becomes rather complicated. The following three characteristic regions are observed in all samples irradiated with a ruby laser using energy fluences in the range 1.0 to $3.0 \mathrm{~J} \mathrm{~cm}^{-2}$ :

(1) A region exterior to the directly irradiated spot which is littered with small, $\sim 0.1 \mu \mathrm{m}$ diameter, carbon spheroids-with a structure similar to that of carbon black. The number density of the spheroids decays with increasing distance from the perimeter of the irradiated region.

(2) A boundary region showing both spheroids and torn layers of carbon, about $1-5 \mu \mathrm{m}$ in lateral extent, on the surface.

(3) A central region with spheroids and surface upheavals which trace out a grain pattern comparable to that of pristine HOPG.

Rippling of the surface was not observed.

The characteristic regions can easily be distinguished with optical microscopy. In Fig. 2(a), an optical micrograph of a sample irradiated with a $2.0 \mathrm{~J} \mathrm{~cm}^{-2}$ pulse, the boundary of the irradiated region is clearly visible. The rough background contrast near the boundary is due to $1-5 \mu \mathrm{m}$ torn layers of carbon resting on the

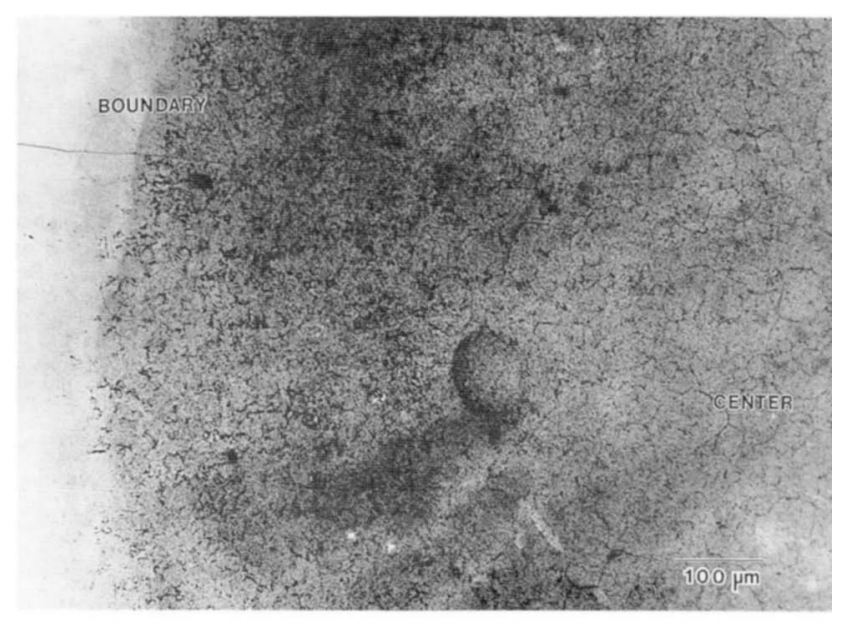

(a)

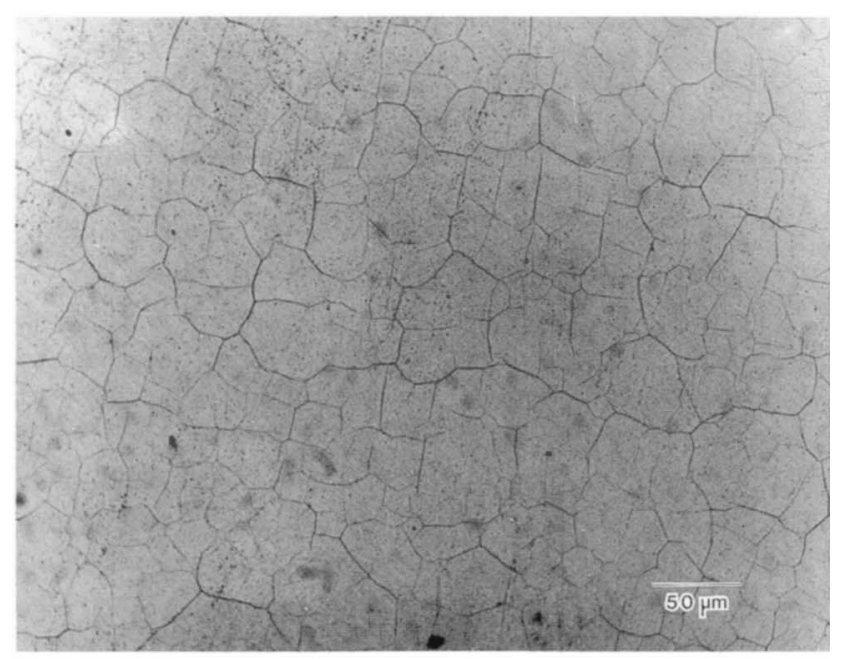

(b)

FIG. 2. Optical micrographs of (a) a sample irradiated with a $2.0 \mathrm{~J} \mathrm{~cm}^{-2} 30 \mathrm{~ns}$ ruby laser pulse and (b) the central region of a sample irradiated with a $3.0 \mathrm{~J} \mathrm{~cm}^{-2} 30 \mathrm{~ns}$ ruby laser pulse. 
surface, whereas the fine background contrast is due to $\sim 0.1 \mu \mathrm{m}$ carbon spheroids on the surface. More detail typical of the central region is shown in Fig. 2(b). Here a grain-defining pattern is observed on the surface. The linear features, as will be shown below, are actually surface upheavals. A more detailed examination provides further structural information on these features.

The torn layers of carbon have maximum density adjacent to the laser irradiated perimeter. The general structural appearance of the torn layers at the boundary is shown by SEM in Figs. 3(a) and 3(b). In Fig. 3(a), it is readily seen that the torn layers are pulled back and elevated from the surface. The torn layer in the lower left-hand corner of Fig. 3(b) shows two important features. The first is that the torn layers are quite thin, at most several hundred $\AA$ thick. Second, spheroids are observed on both sides of the torn layers of carbon. This indicates that the spheroids impacted the layer after it had peeled away from the surface.

Further examination of the spheroids leads to interesting surface morphologies, as shown in Fig. 4. In this instance, in the center of a sample irradiated with a $3.0 \mathrm{~J} \mathrm{~cm}^{-2} 30 \mathrm{~ns}$ ruby laser pulse, there are three spheroids which form a stack in the center of the micrograph. The only plausible explanation for such a morphology is that the carbon in the spheroids has made an excursion into the atmosphere near the irradiated surface prior to reattachment. Small carbon black spheres have frequently been observed near carbon arcs. ${ }^{9}$ A mechanism for the formation of the carbon spheroids is discussed below.

Surface upheavals are the other structural feature of significance in the center of the irradiated samples. These upheavals are clearly observed on the surface of a sample irradiated with a $2.5 \mathrm{~J} \mathrm{~cm}^{-2}$ laser pulse, as shown in Fig. 5. The upheavals are the result of compressive deformation of the substrate, presumably at grain boundaries during rapid thermal expansion.

All of the general features of the structure are seen by TEM and can be directly attributed to the solidification of laser melted carbon. Figure 6, a transmission electron micrograph of the damaged region of a sample irradiated with a $3.0 \mathrm{~J} \mathrm{~cm}^{-2}$ pulse, shows a torn layer, spheroids, and one surface upheaval. Selected area diffraction patterns from areas as small as $1 \mu \mathrm{m}^{2}$ display a fully developed ring pattern, as shown in Fig. 7(a) for a sample irradiated with a $30 \mathrm{~ns} 2.5 \mathrm{~J} \mathrm{~cm}^{-2}$ pulse. The periphery of the bright area surrounding the forward scattered beam corresponds to the 002 ring (we maintain the indexing scheme for graphite - the $c$-axis spacing is equal to twice the interlayer spacing). The outer two rings correspond to 100 and 110 . This diffraction pattern is entirely consistent with previously published work ${ }^{5}$ and corresponds to a randomly oriented 2dimensional carbon structure.
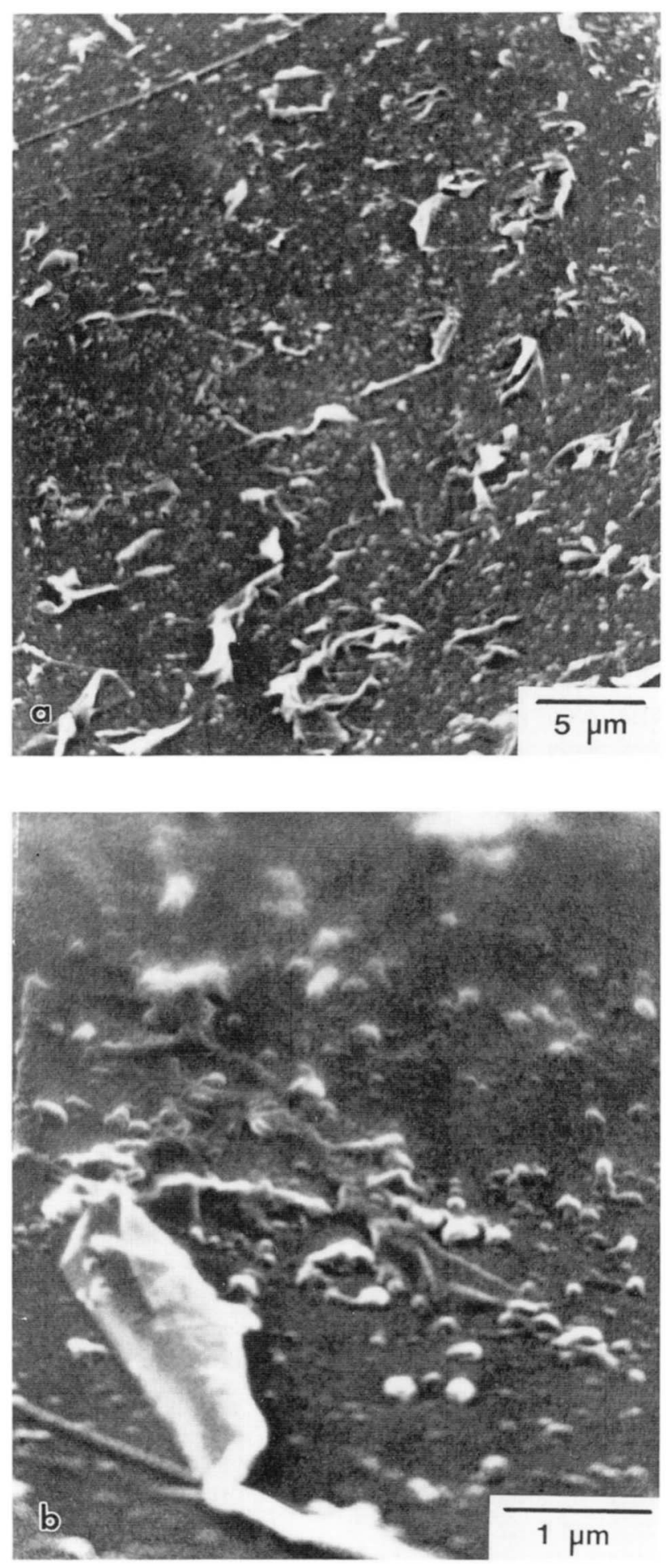

FIG. 3. Scanning electron micrographs of the boundary region of a sample irradiated with a $2.5 \mathrm{~J} \mathrm{~cm}^{-2} 30$ ns ruby laser pulse. (a) Low magnification view showing general structure. (b) High magnification view showing detail of torn layers and spheroids.

The similarity in behavior between nanosecond and picosecond pulsed laser irradiation is verified by electron diffraction studies. In Fig. 7(b) we show a 


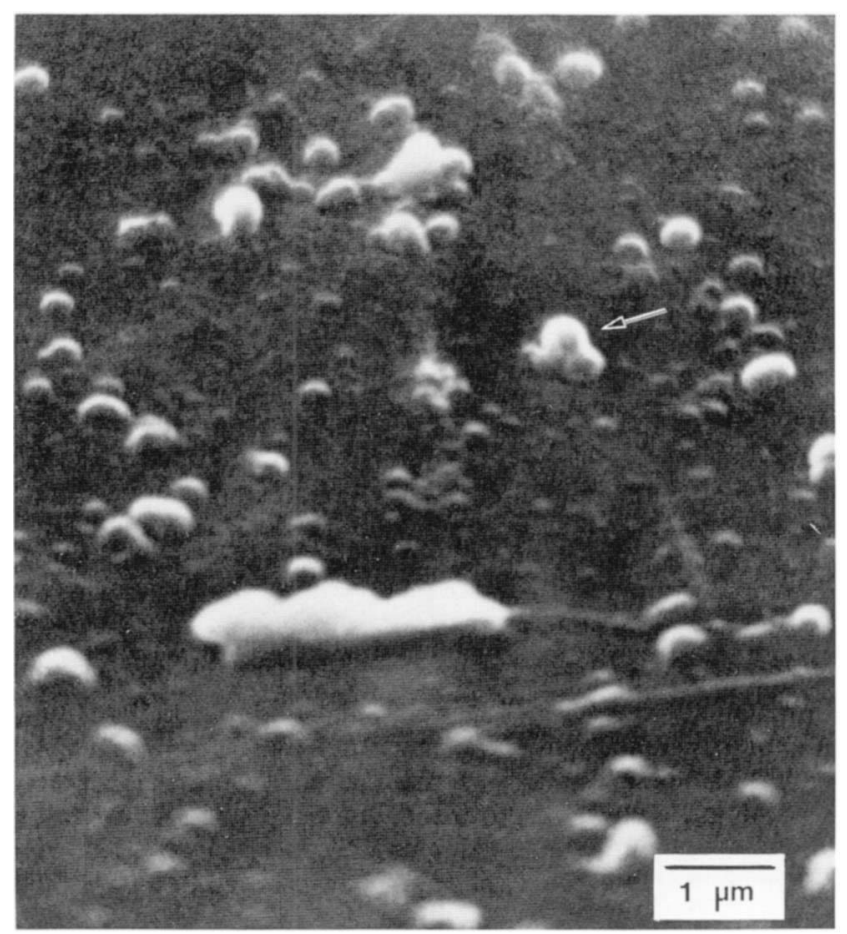

FIG. 4. The surface structure in the central region of a sample irradiated with a $3.0 \mathrm{~J} \mathrm{~cm}^{-2} 30 \mathrm{~ns}$ ruby laser pulse. Notice the stacking of the spheroids in the center of the micrograph (see arrow).

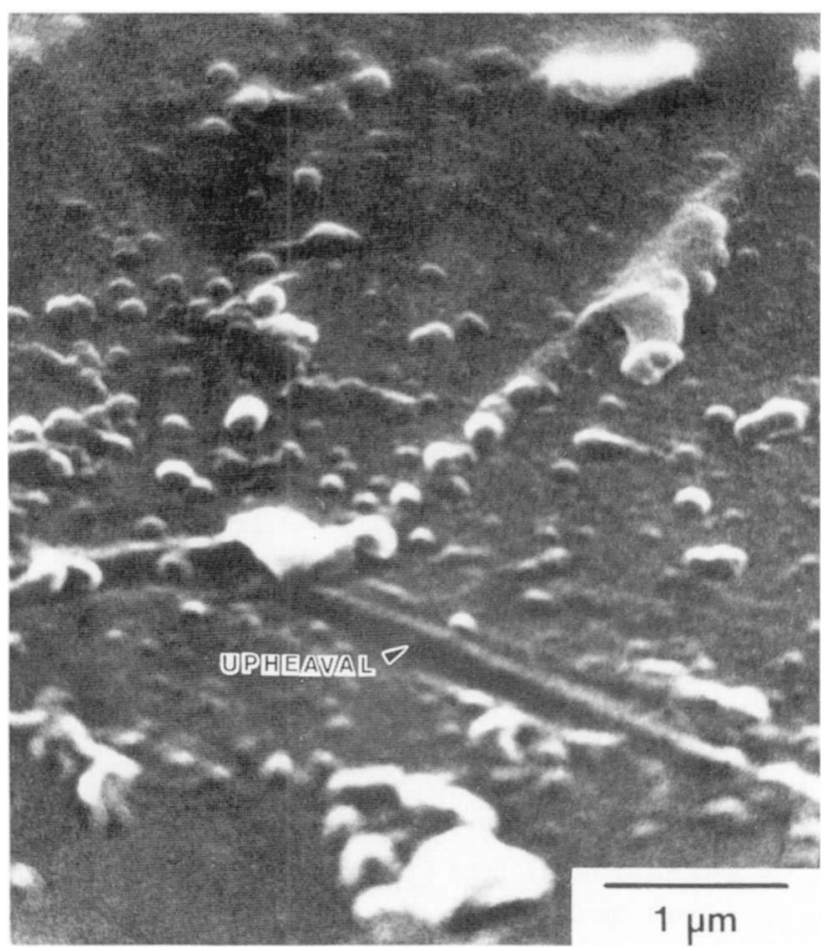

FIG. 5. Scanning electron micrograph of spheroids and surface upheavals in the central region of a sample irradiated with a $2.5 \mathrm{~J} \mathrm{~cm}^{-2} 30 \mathrm{~ns}$ ruby laser pulse. Note that the surface upheavals meet to form an apparent three grain junction in the center of the photograph.

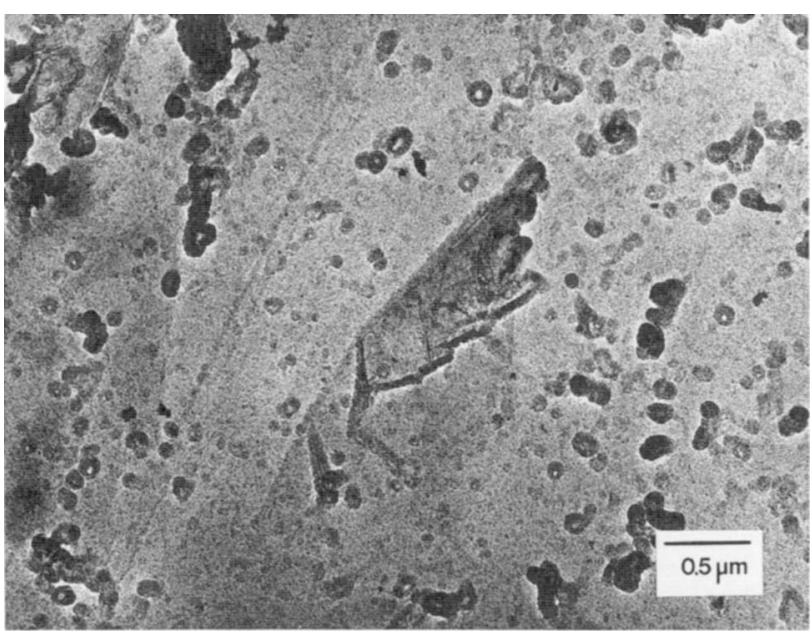

FIG. 6. The general structure, as seen by TEM, of a sample irradiated with a $3.0 \mathrm{~J} \mathrm{~cm}^{-2} 30 \mathrm{~ns}$ ruby laser pulse. $\sim 0.1 \mu \mathrm{m}$ diameter carbon spheroids are randomly dispersed. A torn layer is visible in the center of the micrograph. The trace of a surface upheaval runs approximately vertically on the left-hand side of the micrograph.

selected area diffraction pattern from a sample irradiated with a $20 \mathrm{ps} 1.8 \mathrm{~J} \mathrm{~cm}^{-2}$ pulse from a $\mathrm{Nd}$ :YAG laser. The similarity in diffraction patterns is evident. At high laser fluences, the diffraction rings sharpen and the 002 reflection loses intensity, as shown in Fig. 8 for a sample irradiated with a $30 \mathrm{~ns} 4.0 \mathrm{~J} \mathrm{~cm}^{-2}$ pulse. A detailed discussion of the diffraction patterns is given below.

It is possible to directly image the grain structure of the resolidified material for cases in which the laser fluence is near that for which the melt depth saturates (see discussion below). A dark-field image formed from a small portion of the 002 ring is shown in Fig. 9 for a sample irradiated with a $20 \mathrm{ps} 1.8 \mathrm{~J} \mathrm{~cm}^{-2}$ pulse at $\lambda=5320 \AA$. The resolidified grains have the morphology of thick disks. The grain size is $\sim 100 \AA$ in the adirection and $\sim 50 \AA$ in the c-direction, as shown in Fig. 9. A grain morphology of this nature is consistent with surface energy minimization. Efforts to image the grain structure in samples irradiated with ruby laser fluences less than $3.0 \mathrm{~J} \mathrm{~cm}^{-2}$ were unsuccessful; only a continuous background contrast was observed. Additionally, all efforts to image the grain structure in dark field using either the 100 or 110 peak were unsuccessful.

\section{DISCUSSION}

In this section qualitative models will be given for the main structural features as they evolve during heating from laser irradiation. Additionally, a detailed discussion of the diffraction patterns will be given at the end of this section.

Periodic rippling of the surface of many laser irradiated materials has been reported. ${ }^{10-12}$ Sipe $e t$ al. ${ }^{13}$ de- 


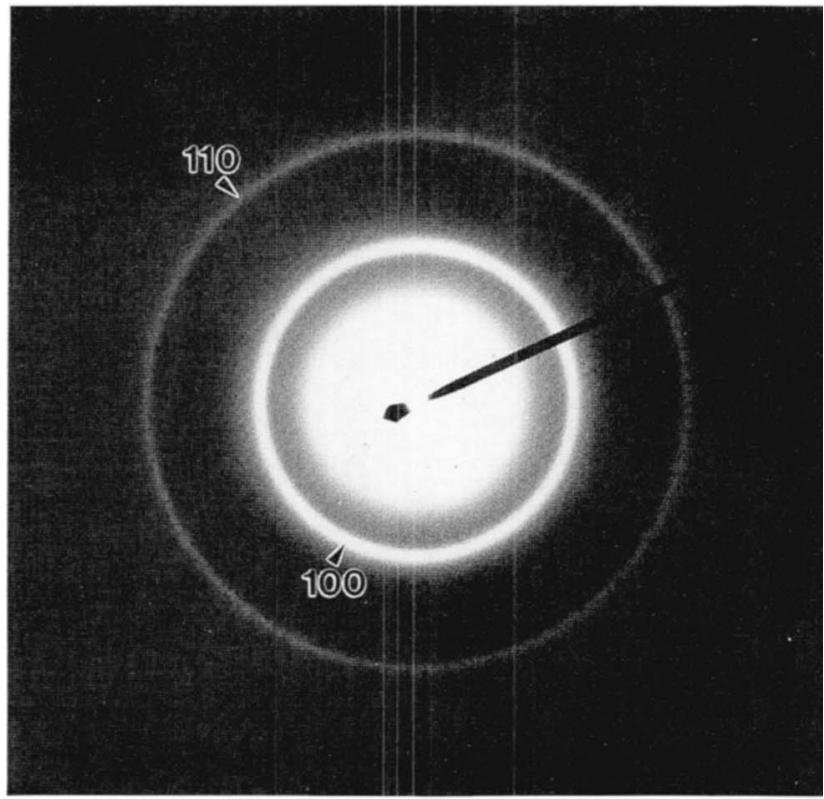

(a)

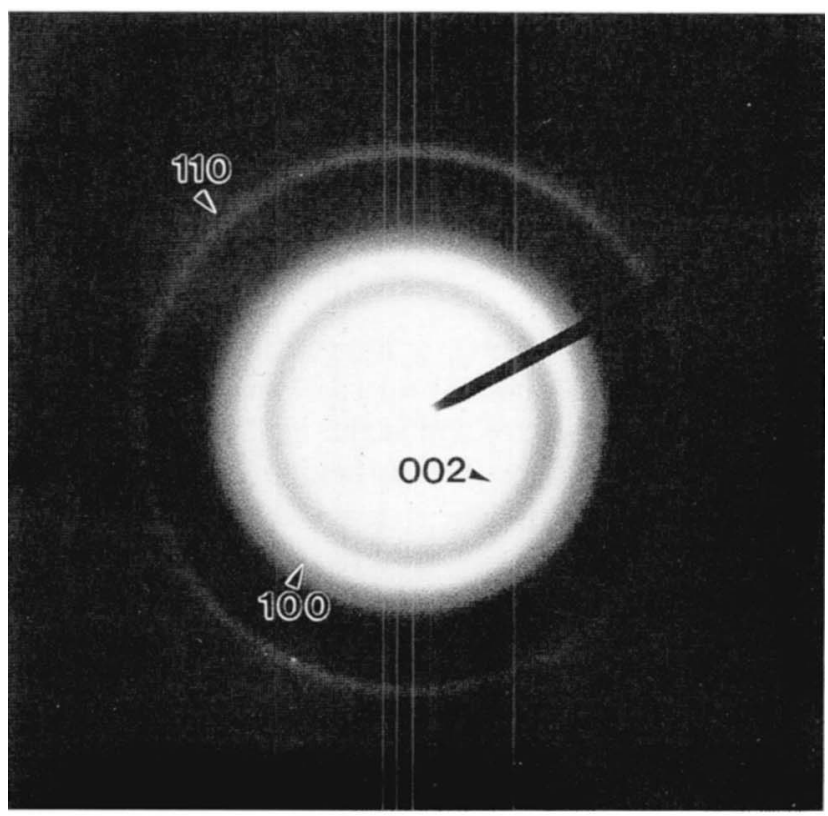

(b)

FIG. 7. Selected area electron diffraction patterns from (a) a sample irradiated with a $2.5 \mathrm{~J} \mathrm{~cm}^{-2} 30 \mathrm{~ns}$ ruby laser pulse $(\lambda=6943 \AA)$ and (b) a $1.8 \mathrm{~J} \mathrm{~cm}^{-2} 20 \mathrm{ps} \mathrm{Nd:YAG} \mathrm{laser} \mathrm{pulse}(\lambda=5320 \AA)$.

veloped a theory to explain surface rippling in terms of inhomogeneous energy deposition of polarized incident radiation. However, we used unpolarized radiation. Hence these effects are not expected nor observed.

\section{A. Surface upheavals and torn layers}

The pattern of surface upheavals in the microstructure shown in Fig. 2(b) strongly suggests the outline of

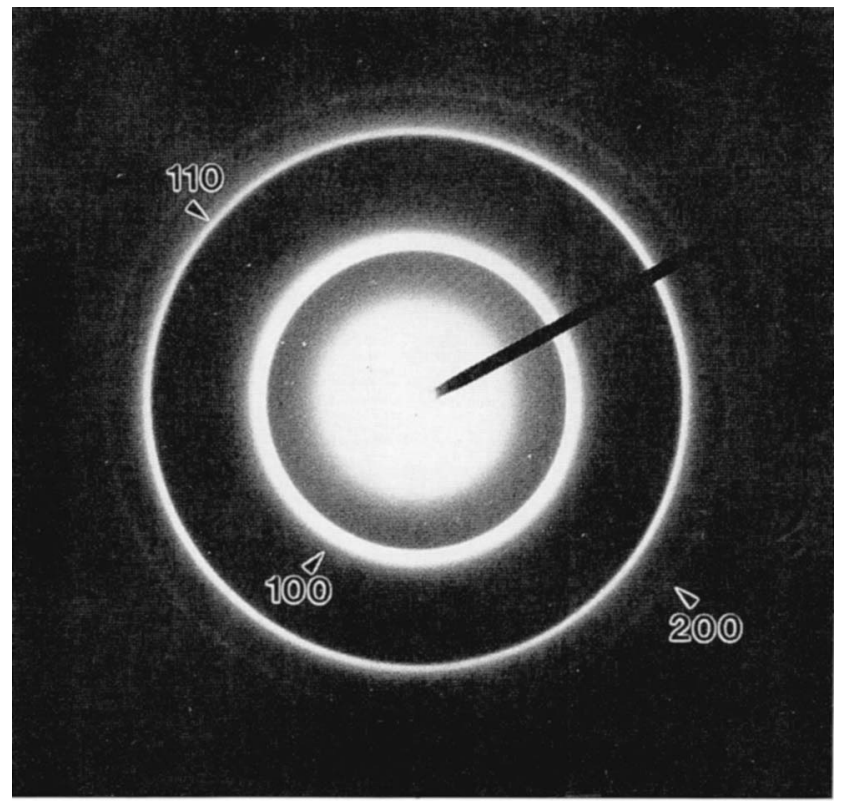

FIG. 8. Selected area electron diffraction pattern from a sample irradiated with a $30 \mathrm{~ns} 4.0 \mathrm{~J} \mathrm{~cm}^{-2}$ ruby laser pulse.

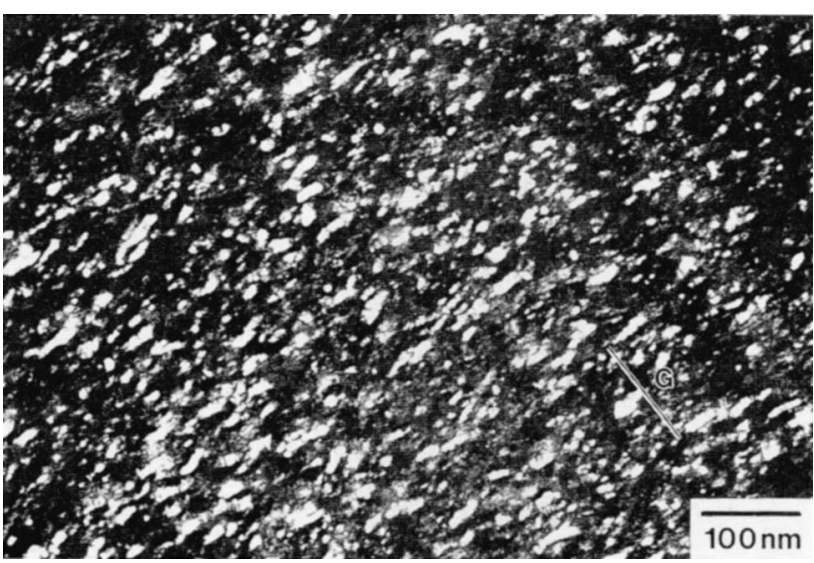

FIG. 9. 002 dark-field image of the damaged region of a sample irradiated with a $1.8 \mathrm{~J} \mathrm{~cm}^{-2} 20 \mathrm{ps} \mathrm{Nd}$ : YAG laser pulse. The image was formed using $0.6 / 2 \pi$ of the 002 ring. $G$ is the direction between 000 and the center of the portion of the 002 ring used to form the dark-field image.

the underlying grain structure of the HOPG. This is additionally suggested by the junction of three upheavals shown in Fig. 5. It is likely that these upheavals originate from compressive stresses in the substrate layers immediately below the melted region of carbon. This is due to heating of the substrate below the thin melted layer of carbon. However, the substrate in the region surrounding the melt pool is near room temperature. Hence the substrate outside of the irradiated area acts as a rigid barrier to thermal expansion of the substrate beneath the melted region of carbon (called constrained inertial confinement ${ }^{14}$ ). The substrate material 
under the beam tries to expand, but it is constrained by the unirradiated material. Hence compressive stresses increase until the material yields. These ideas are schematically depicted in the left panel and the upper part of the right panel of Fig. 10.

The tearing of the surface of the resolidified material, for example shown in Fig. 3, is attributed to thermal contraction of the resolidified material and the substrate. Hence, this is opposite to the situation described above. In this case, however, tearing can occur both in the substrate and in the resolidified material. This process is schematically depicted in the lower part of the right panel on Fig. 10.

The ideas of thermomechanical degradation of pulse irradiated surfaces have been worked out by Musal for elastically isotropic surfaces. ${ }^{15}$ Musal solved the governing equations for the case of transverse inertial confinement. The minimum temperature rise necessary to give plastic deformation was determined to be

$$
\Delta T=\frac{(1-\nu)}{E \alpha} Y
$$

where $\nu$ is Poisson's ratio, $E$ is Young's modulus, $Y$ is the yield stress of the material, and $\alpha$ is the linear coefficient of thermal expansion. Musal determined that the heated surface can be approximated by a state of planestress. Hence this analysis is also applicable to laserpulsed graphite when the incident beam is normal to the graphite basal planes. For an order of magnitude estimate of the temperature rise necessary to cause deformation in the graphite, we use the theoretical in-plane tensile strength $(Y)$ and Young's modulus $(E)$ for single crystal graphite; $E=\sim 1000 \mathrm{GPa}$ and $Y=20 \mathrm{GPa}^{16}$ The in-plane thermal expansion coeffi- cient for graphite shows anomalous behavior. For temperatures in the range $0-750 \mathrm{~K}, \alpha$ is negative, while for temperatures greater than $\sim 1000 \mathrm{~K}, \alpha$ saturates at $\sim 1 \times 10^{-6} \mathrm{~K}^{-1} .{ }^{17}$ Since we are interested in the behavior of the substrate up to temperatures of $\sim 4500 \mathrm{~K}, \alpha$ will be approximated to be $\sim 1 \times 10^{-6} \mathrm{~K}^{-1}$. If we approximate Poisson's ratio to be 0.5 , we find that the temperature rise necessary to cause plastic deformation is $\sim 10000 \mathrm{~K}$. If we use a more reasonable value for the in-plane tensile strength reported to be $2 \mathrm{GPa}^{18}$ (attributable to defects in the HOPG), we find the temperature rise necessary to cause deformation to be $\sim 1000 \mathrm{~K}$. We know that there is at least a $3000-4000 \mathrm{~K}$ temperature rise because the sample melts. Hence, it is reasonable to interpret both the surface upheavals and surface tears as thermal stress-induced features. (Surprisingly, the temperature rise necessary to cause plastic deformation in annealed pure $\mathrm{Cu}$ is only $20 \mathrm{~K} .{ }^{15}$ This is due to the low yield stress in pure $\mathrm{Cu}$ crystals.)

\section{B. Carbon spheroids and hydrodynamic sputtering}

Perhaps the most interesting surface features are the small carbon spheroids. It is clear from the SEM results shown in Fig. 4 that the carbon spheroids have been ejected from the surface, only to return later either by gravitational or electrostatic attraction. Similar structures have been observed in laser-melted surfaces of $\mathrm{Bi}$ and $\mathrm{PbTe},{ }^{19} \mathrm{Cu},{ }^{20}$ and $\mathrm{Si}^{21}$ Recently a theory has been developed to explain the presence of small sputtered spheres of material on laser-irradiated surfaces. ${ }^{14}$

The theory of hydrodynamic sputtering developed by Kelly and Rothenberg ${ }^{14}$ assumes that there are in-

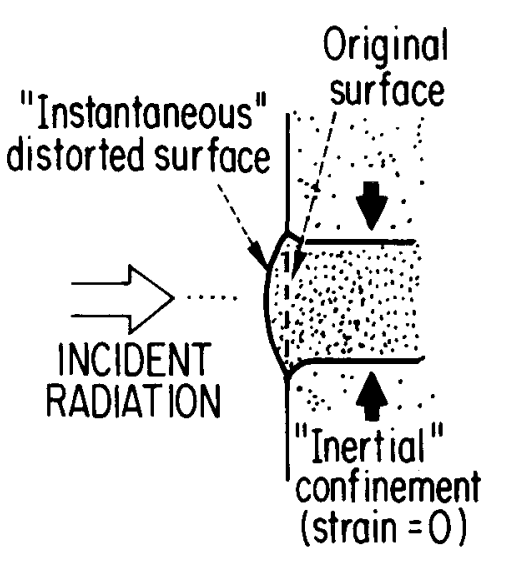

Side view

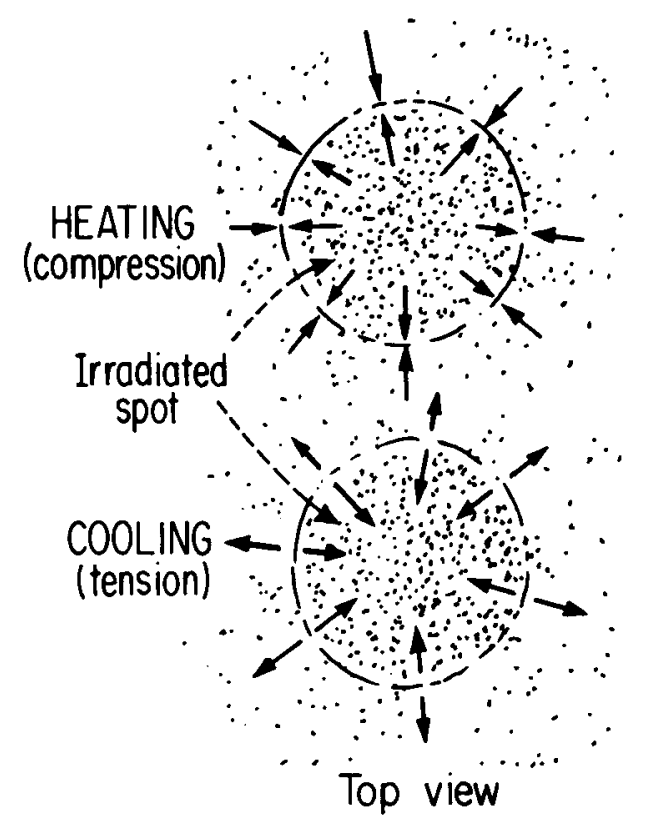

FIG. 10. Irradiated surface thermal distortion and thermomechanical degradation. (Taken from Musal ${ }^{15}$ ) 
homogeneities (called asperities) on the surface of the solid substrate prior to laser irradiation, shown schematically in Fig. 11(a). During laser irradiation there is an additional expansion $\Delta L$ of the asperity relative to the molten substrate. This expansion imparts a momentum to the particle $\left(4 \pi r^{3} \rho_{l} / 3\right)(\Delta L / \Delta t)$, where $\Delta t$ is the duration of the pulse, $\Delta L=2 r \alpha \Delta T+$ $2 r\left[\left(\rho_{s}-\rho_{l}\right) / 3 \rho_{s}\right]$, in which $\alpha$ is the linear thermal expansion coefficient, $\rho_{s}$ is the solid density, and $\rho_{l}$ is the liquid density. The formation of the particle is opposed by a surface tension force $f=-\left[\partial\left(4 \pi r^{2} \gamma\right) / \partial r\right]=-8 \pi r \gamma$, where $\gamma$ is the surface energy of the liquid. Completing the momentum balance, Kelly and Rothenberg derived an expression for the minimum droplet size, $r_{\min }$, which can be expelled from the substrate:

$$
r_{\min } \geqslant\left[\left(\frac{3 \gamma}{\rho_{l}(\Delta L / L)}\right)(\Delta t)^{2}\right]^{1 / 3}
$$

Numerical evaluation of this expression is difficult because of our ignorance of the magnitude of many of the parameters of liquid carbon (such as surface energy, linear expansion coefficient, density, and overheating in the asperity). The important point, however, is that this expression does predict a minimum size of the expelled spheroids. Examination of the TEM and SEM micrographs shows that the ejected spheroids have a minimum size of $\sim 0.1 \mu \mathrm{m}$, which supports the theoretical idea of Kelly and Rothenberg that there is a minimum size for the ejected particle.

\section{Electron scattering and imaging}

The nature of the diffraction patterns from the irradiated material will now be considered. A calculated electron diffraction pattern from a powder sample of fully graphitized carbon is shown in Fig. 12. Excluding 002 , it is seen that the $h k l$ reflections with the greatest intensity are 101 and 112 . The diffraction patterns for the laser-irradiated material shown in Figs. 7 and 8

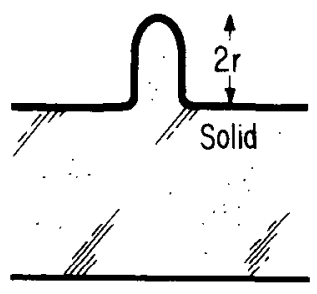

(a)

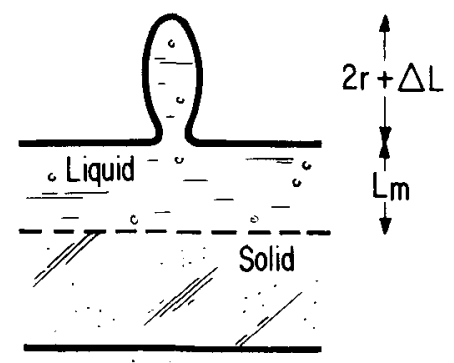

(b)
FIG. 11. Sketch relevant to hydrodynamical sputtering. (a) A surface asperity with height $2 r$. (b) The same asperity pulling away from the laser irradiated surface. $\Delta L$ is the additional thermal expansion of the asperity relative to the molten substrate. $L_{m}$ is the surface melt depth. (Taken from Kelly and Rothenberg ${ }^{14}$ )

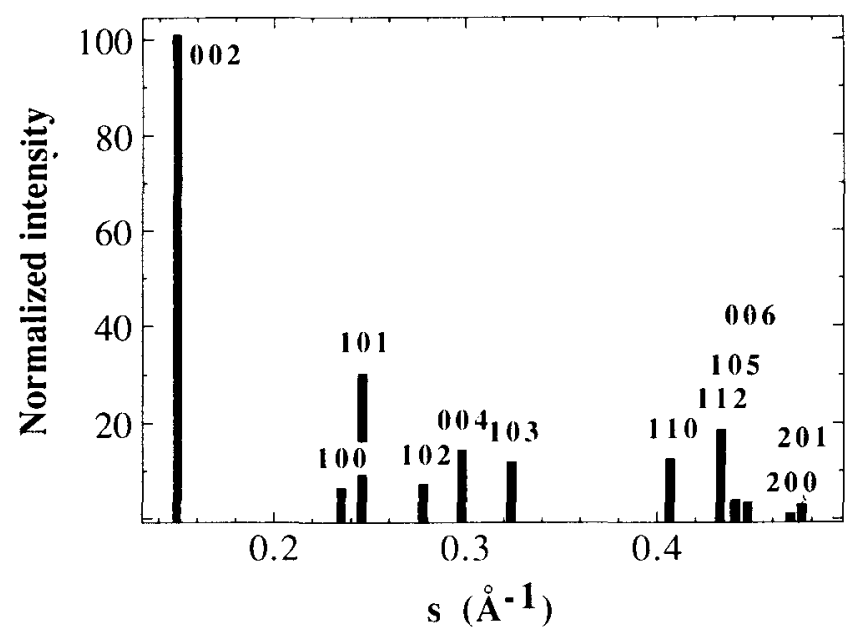

FIG. 12. Calculated ring electron diffraction pattern for fully crystalline graphite.

display a 002 ring and broad 100 and 110 bands. This result suggests that the resolidified carbon is only turbostratically ordered (random stacking of the layers). The $h k$ component of a powder pattern of a turbostratic carbon consists of a sharp rise in intensity on the low $s$ side of the peak $(s=\sin \theta / \lambda)$, and then a long tail on the high $s$ side of the peak which decreases approximately as $f^{2} / s^{2}$, where $f$ is the atomic scattering factor for carbon. An idealized pattern is shown in Fig. 13. Detailed discussions of such diffraction patterns are given elsewhere. ${ }^{22,23}$

The nature of the diffraction patterns can be illuminated by performing microdensitometer measurements of the scattered intensity. The scattered intensity is proportional to the photographic density $D=$ $\log (1 / T)$, where $T$ is the fraction of light transmitted through the negative. ${ }^{24}$ The radial dependence of the intensity for several irradiated samples is given in Fig. 14. Measuring the 002 peak is difficult due to its proximity to the forward scattered beam. What we do find are broad bands for the 100 and 110 peaks. No 004 peak is discernible. The absence of a 004 peak indicates that there is some alignment of the $c$-axes of the resolidified grains with the $c$-axis of the substrate (the beam direction is coincident with $c$-axis of the substrate). This is not surprising since the interfacial energy of the

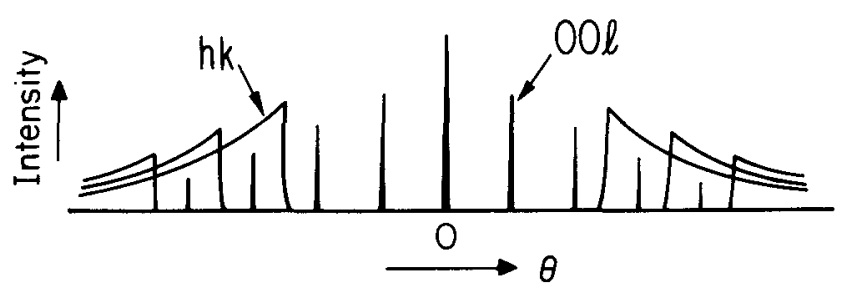

FIG. 13. Schematic powder diffraction pattern expected from a turbostratic material. (Taken from Cowley ${ }^{22}$ ) 


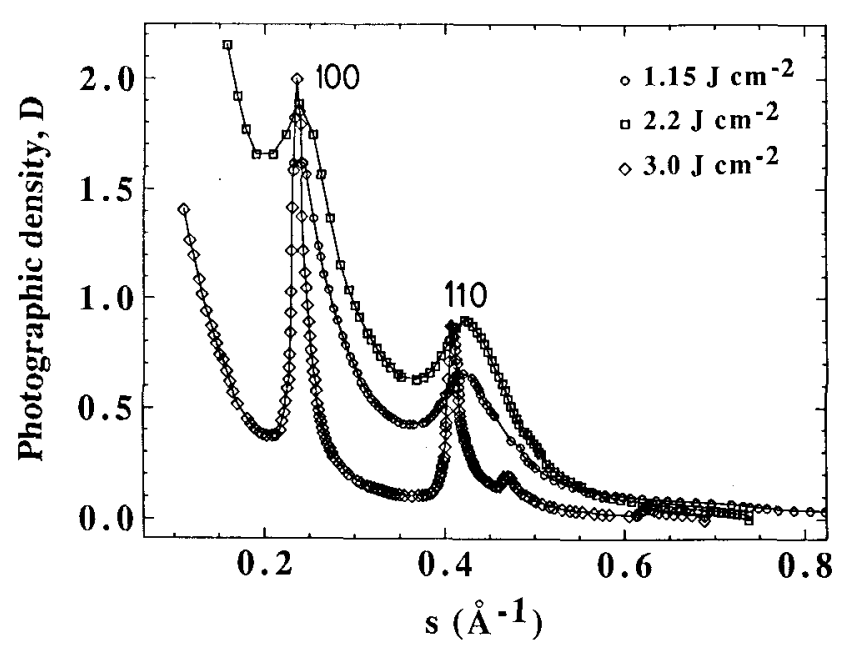

FIG. 14. Scattered electron intensity as a function of $s$ (where $s=$ $\sin \theta / \lambda, \AA^{-1}$ ) for samples irradiated with $1.15,2.2$, and $3.0 \mathrm{~J} \mathrm{~cm}^{-2}$ $30 \mathrm{~ns}$ ruby laser pulses.

resolidified grains is minimized by increasing the alignment of their $c$-axes with the $c$-axis of the substrate.

The widths of the $h k$ peaks shown in Fig. 14 are inversely proportional to the average in-plane crystallographic coherence length $L_{a}{ }^{23,25}$ If there is complete alignment of the $c$-axes of the resolidified material and this direction is parallel to the incident beam direction, then the expression for $L_{a}$ is the same as that for the fully crystalline material, namely the Scherrer equation:

$$
L_{a}=\frac{0.94 \lambda}{B \cos \theta}
$$

where $\lambda$ is the electron wavelength, $\theta$ is the Bragg angle, and $B$ is the FWHM peak breadth in radians. If, however, the orientation of the resolidified material is completely random, then $L_{a}$ is given as ${ }^{23}$ :

$$
L_{a}=\frac{1.84 \lambda}{B \cos \theta} \text {. }
$$

Since we observe no 004 peak, the numerical coefficient we use should be an intermediate value between 0.94 and 1.84. Stating this, Eq. (4) is chosen to calculate $L_{a}$ values, since a larger numerical coefficient partially corrects for instrumental broadening of the peaks. An $L_{a}$ value of $-20 \AA$ is calculated from the 110 peak of the samples irradiated with 1.15 and $2.2 \mathrm{~J} \mathrm{~cm}^{-2} 30 \mathrm{~ns}$ ruby laser pulses. An $L_{a}$ value of $\sim 70 \AA$ is calculated for the sample irradiated with a $3.0 \mathrm{~J} \mathrm{~cm}^{-2} 30 \mathrm{~ns}$ ruby laser pulse. These results are in agreement (within a factor of 2) with grain sizes determined by Raman spectroscopy, ${ }^{26}$ as shown in Fig. 15. The diffraction results show that the liquid carbon resolidifies with a finegrained turbostratic microstructure.

The dark-field imaging results, such as those shown in Fig. 9, also give good agreement with the average

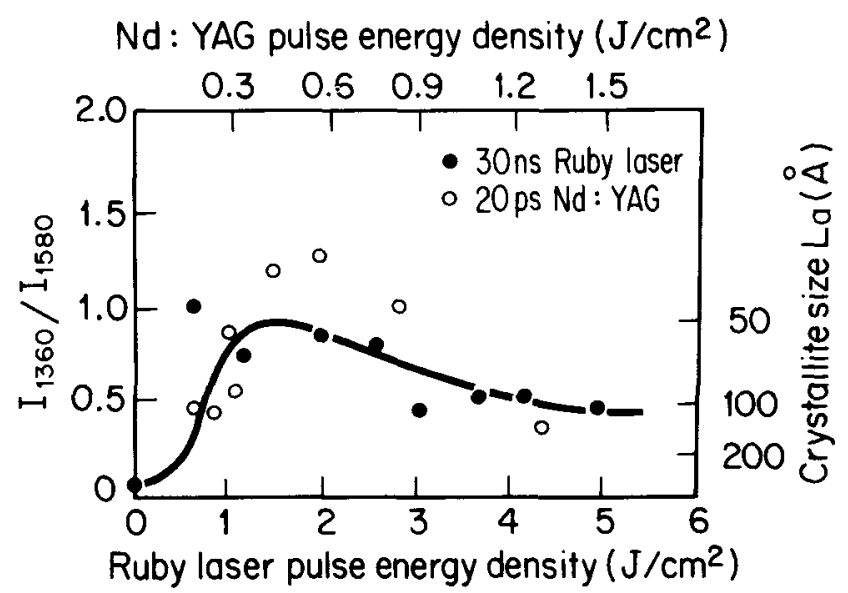

FIG. 15. Raman intensity ratio $I_{1360} / I_{1580}$ for laser irradiated graphite as a function of laser energy density for 30 ns ruby laser pulses (lower abscissa) and 20 ps Nd:YAG laser pulses (upper abscissa). The abscissas in the figure have been set so that comparisons between the two irradiating conditions can be made easily. The solid line is a guide to the eye. (Taken from Steinbeck et al. ${ }^{27}$ )

grain size measured by electron diffraction and Raman spectroscopy. For the sample irradiated with a $1.8 \mathrm{~J} \mathrm{~cm}^{-2}$ $20 \mathrm{ps}$ laser pulse, $L_{a}$ measured from the 002 darkfield image is $\sim 100 \AA$. Referring to Fig. 15, we see that the grain size determined from Raman spectroscopy is extrapolated to be on the order of $100 \AA$.

The diffraction measurements show that the relative intensity of the 002 ring decreases with increasing pulse fluence, indicating that the $c$-axis alignment of the resolidified material increases with laser fluence. At low pulse fluences, the melt pool is shallow and just above the melting temperature; hence the material resolidifies with a random orientation. At high pulse fluences, the melt pool deepens, and the average liquid temperature rises; hence there is more time for alignment of the $c$-axis of the resolidified material with the substrate $c$-axis. The melt depth increases with pulse fluence up to a saturation value, as shown in Fig. 1. Efforts to image the resolidified grain structure at low pulse fluences were unsuccessful because of the high degree of randomness of the structure and the small grain size. For example, a sample irradiated with a $1.15 \mathrm{~J} \mathrm{~cm}^{-2} 30 \mathrm{~ns}$ ruby laser pulse will have an $L_{a}$ value of $\sim 20 \AA$. The thickness of the foil used for the microscopy experiment is $\sim 1000 \AA$ (the value of the disorder depth determined by RBS, as shown in Fig. 1). Hence there are $\sim 50$ grains through the thickness of the foil. To form a quasi-continuous intensity dark-field image, 10 out of 50 of these grains need to have 002 in the vicinity of the Ewald sphere (the factor of 10 comes from the fact that the objective aperture used for these experiments encompasses $\sim 10 \%$ of the 002 ring). However, a sample irradiated with a $4.0 \mathrm{~J} \mathrm{~cm}^{-2} 30 \mathrm{~ns}$ ruby laser pulse will have an $L_{a}$ value of $\sim 100 \AA$. The thickness of the foil is $\sim 2000 \AA$. In this case there are $\sim 20$ 
grains through the thickness of the foil. Hence it would be necessary for half of the grains to have 002 near the Ewald sphere to form a continuous dark-field image. The diffraction results for the $4.0 \mathrm{~J} \mathrm{~cm}^{-2}$ pulse show that perhaps $<1 \%$ of the grains have 002 near the Ewald sphere (Fig. 8). Hence it is in the high energy density regime that the grains oriented to diffract strongly are sufficiently spaced to be easily resolved in dark-field images, such as that shown in Fig. 9. The failure to resolve the grain structure in nanocrystalline materials lies in the fact that the dark-field images from these materials frequently give too much information to be resolved, that is to say, that there is sufficient overlap of properly oriented grains to give approximately a continuous intensity level in the dark-field images.

\section{CONCLUSIONS}

(1) The upheavals observed on the surface of the irradiated region are attributed to compressive failures of the substrate during the heating. Similarly, the surface tearing is attributed to tensile failure of both the resolidified material and the substrate during postirradiation cooling. Using measured values for the basal plane tensile strength of graphite, it is estimated that a temperature change of $\sim 1000 \mathrm{~K}$ in the substrate or resolidified layer will cause sufficient thermal stress for tearing to occur.

(2) The small carbon spheroids which litter the surface of the irradiated region are due to hydrodynamic sputtering of surface asperities. The minimum size of the spheroids on the laser-irradiated surfaces was $\sim 0.1 \mu \mathrm{m}$. This size is comparable to that on surfaces of other laser-irradiated materials.

(3) There is increasing alignment of the $c$-axes of the resolidified grains with the $c$-axis of the substrate as the laser pulse fluence increases. The resolidified material is turbostratically ordered. The basal plane grain size of the resolidified material varies from $\sim 20 \AA$ for samples irradiated with fluences just above the melt threshold to $\sim 100 \AA$ for samples irradiated with high fluences. Consistent results are obtained between Raman spectroscopy, electron diffraction, and TEM imaging in determining the grain size of the resolidified material.

(4) The dark-field images of the resolidified material demonstrate the possibility of imaging nanocrystalline materials with favorable grain textures.

\section{ACKNOWLEDGMENTS}

We would like to thank Dr. G. Dresselhaus for many useful discussions. The contributions of Dr. G. Braunstein of Kodak Research Labs, Dr. C.Y. Huang of
Los Alamos National Laboratory, and Dr. T. Venkatesan of the Bellcore Research Labs are greatly appreciated. This work has been supported under AFOSR contract No. F49620-85-0147.

\section{REFERENCES}

${ }^{1}$ F. P. Bundy, in Solid State Physics Under Pressure, edited by S. Minomura (Reidel, New York, 1986), p. 1.

${ }^{2}$ J. Steinbeck, Studies of the High Temperature Properties of Graphite and Liquid Carbon Using Pulsed Laser Heating (Ph.D. Thesis, MIT, 1987).

${ }^{3}$ F. P. Bundy, J. Chem. Phys. 38, 618 (1963).

${ }^{4}$ J. Heremans, C. H. Olk, G. L. Eesley, J. Steinbeck, and G. Dresselhaus, Phys. Rev. Lett. 60, 452 (1988).

${ }^{5}$ T. Venkatesan, D.C. Jacobson, J. M. Gibson, B. S. EIman, G. Braunstein, and M.S. Dresselhaus, Phys. Rev. Lett. 53, 360 (1984).

${ }^{6}$ A. M. Malvezzi, N. Bloembergen, and C.Y. Huang, Phys. Rev. Lett. 57, 146 (1986).

${ }^{7}$ D. H. Reitze, X. Wang, H. Ahn, and M. C. Downer, Phys. Rev. B 40, 11986 (1989).

${ }^{8}$ G. Braunstein, J. Steinbeck, M. S. Dresselhaus, G. Dresselhaus, B.S. Elman, T. Venkatesan, B. Wilkens, and D. C. Jacobson (Proc. Mater. Res. Soc. Symp.) (Materials Research Society, Pittsburgh, PA, 1986), Vol. 51, p. 233.

9J. Abrahamson, Carbon 12, 111 (1974).

${ }^{10}$ P. M. Fauchet and A. E. Siegman, Appl. Phys. Lett. 40, 824 (1982).

${ }^{11}$ J. F. Young, J. S. Preston, H. M. van Driel, and J. E. Sipe, Phys. Rev. B 27, 1155 (1983).

${ }^{12}$ I.W. Boyd, S. C. Moss, T. F. Boggess, and A. L. Smirl, Appl. Phys. Lett. 45, 80 (1984).

${ }^{13}$ J. E. Sipe, J. F. Young, J. S. Preston, and H. M. van Driel, Phys. Rev. B 27, 1141 (1983).

${ }^{14}$ R. Kelly and J. E. Rothenberg, Nucl. Instr. Meth. Phys. B7/8, 755 (1985).

${ }^{15}$ H. M. Musal, Symp. on Optical Materials for High Power Lasers, Boulder, CO, 159 (1979).

${ }^{16}$ M. S. Dresselhaus, G. Dresselhaus, K. Sugihara, I. L. Spain, and H. A. Goldberg, Graphite Fibers and Filaments (Springer-Verlag, New York, 1988).

${ }^{17}$ B.T. Kelly, Physics of Graphite (Applied Science, London, 1981).

${ }^{18}$ G. G. Tibbetts and C. P. Beetz, J. Phys. D 20, 292 (1987).

${ }^{19}$ S.V. Gaponov, A. A. Gudkov, and A. A. Fraerman, Sov. Tech. Phys. 27, 1130 (1982).

${ }^{20}$ S. J. Thomas, R. F. Harrison, and J. F. Figueira, Appl. Phys. Lett. 40, 200 (1982).

${ }^{21}$ J. E. Rothenberg and R. Kelly, Nucl. Instr. and Meth. B1, 291 (1984).

${ }^{22}$ J. M. Cowley, Diffraction Physics (North-Holland, New York, 1975).

${ }^{23}$ B. E. Warren, Phys. Rev. 59, 693 (1941)

${ }^{24}$ M. J. Buerger, Crystal Structure Analysis (Wiley, New York, 1960).

${ }^{25}$ B. E. Warren, X-Ray Diffraction (Addison-Wesley, Reading, MA, 1969).

${ }^{26}$ M. S. Dresselhaus and J. Steinbeck, Tanso 132, 44 (1988).

${ }^{27}$ J. Steinbeck, G. Braunstein, J. Speck, M. S. Dresselhaus, C.Y. Huang, A. M. Malvezzi, and N. Bloembergen (Proc. Mater. Res. Soc. Symp.) (Materials Research Society, Pittsburgh, PA, 1987), Vol. 74, p. 263. 